\title{
Transarticular external fixation versus deltoid ligament repair in treating SER IV ankle fractures: a comparative study
}

\author{
Bohua Li ${ }^{1,2}$, Shanxi Wang ${ }^{2}$, Zhengdong Zhang ${ }^{2}$, Hai Yang ${ }^{2}$, Jun Li ${ }^{2}$, Qin Li ${ }^{2}$ and Lei Liu ${ }^{2 *}$
}

\begin{abstract}
Background: The topic that whether the injured deltoid ligament should be repaired when associated with ankle joint fractures is still discussed. The objective of this study was to compare the clinical effect of open reduction and internal fixation (ORIF) with deltoid ligament repair (DLR) or transarticular external fixation (TEF) in treating supination-external rotation type IV (SER IV) ankle fractures.

Methods: Between January 2012 and December 2015, 43 patients were diagnosed as SER IV ankle fractures, 20 underwent ORIF and transarticular external fixation (TEF) without DLR (group 1), 23 were treated with ORIF and DLR (group 2). The pre- and post-operative radiographic examination were performed, the American Orthopaedic Foot and Ankle Society (AOFAS) ankle-hindfoot score, the visual analog scale (VAS), the Medical Outcomes Short Form 36-item questionnaire score (SF-36), and the ankle range of motion (ROM) were used for functional evaluation.

Results: In both groups, the three scores improved significantly after surgery, but there was no significant difference between the two groups. At 6 weeks after surgery, patients in group 2 had better ankle ROM than group 1 (29.35 \pm 2.033 vs. $40.35 \pm 3.550, P<0.001)$, but there was no difference at 12 months postoperatively. No cases of bone nonunion or post-traumatic arthritic changes were seen during the follow-up. Patients in group 1 required a shorter time to achieve fracture union than patients in group 2.

Conclusions: ORIF with TIF is an optional strategy to manage SER IV ankle fractures as it achieves comparable functional results to ORIF with DLR. It also allows patients to start relatively earlier weight-bearing and may promote fracture union.
\end{abstract}

Keywords: Transarticular external fixation, ORIF, Deltoid ligament repair, Supination-external rotation, Ankle fracture

\section{Background}

The deltoid ligament is an important structure to stabilize the ankle and maintain the normal anatomical position of the talus, inappropriate treatment for the ruptured deltoid ligament may cause ankle instability and traumatic arthritis [1-4]. Supination external rotation type IV (SER IV) ankle fractures are one of the most common and unstable fractures in the clinical which need surgical treatment [3,5-9]. When the medial malleolus is intact, this type of fractures represents the ones with the injury of the deltoid ligament (AO/OTA

\footnotetext{
* Correspondence: liuinsistence@163.com

${ }^{2}$ Department of Orthopedics, West China Hospital, Sichuan University, 37\#

Wainan Guoxue Road, Chengdu 610041, People's Republic of China

Full list of author information is available at the end of the article
}

classification 44-B3.1). Whether the ruptured deltoid ligament should be repaired or not is still controversy.

In recent years, suture-anchor was widely used for patients treated with deltoid ligament repair (DLR) [9-11], and plaster casts were most often used in treatment without DLR. Previous studies had shown that transarticular external fixators might raplace the plaster casts well by offering the opportunity for self-healing of deltoid ligament and allowing early weight bearing [12-14]. Our previous study had also shown that open reduction and internal fixation (ORIF) with transarticular external fixation (TEF) but no DLR could achieve satisfactory outcomes in treating SER IV ankle fractures [15].

Up to now, studies about ORIF, TEF but no DLR versus ORIF and DLR in the treatment of SER IV ankle 
fractures are rare. In this study we focused to compare the clinical and radiographic outcomes of these two methods.

\section{Methods \\ Patients}

This study was approved by the internal research board of our hospital. We retrospectively reviewed patients who were diagnosed as SER IV ankle fractures (AO/ OTA classification 44-B3.1), they underwent surgical treatment between January 2012 and December 2015. The inclusion criteria were as follows: patients underwent ORIF with TEF or ORIF with DLR, pre- and postoperative $\mathrm{X}$-rays evaluation, a preoperative magnetic resonance imaging (MRI) and three-dimensional computed tomography $(\mathrm{CT})$, patients greater than 16 years old, and at least 12 months follow-up. The exclusion criteria included medial malleolar fracture with deltoid ligament rupture, multiple fractures, concomitant ligament injuries, open fractures, old fractures, pathological fractures, severe systemic illness, preoperative ankle dysfunction.

A total of 745 ankle fractures treated operatively were identified initially. 584 were excluded because of multiple and open fractures, 23 old fractures were also excluded, 78 were excluded because of no MRI examination, 17 were excluded because of loss of followup. Finally, 43 patients were incorporated into the study cohort, among which 20 were treated with ORIF and TEF (group 1), 23 were treated with ORIF and DLR (group 2). The patients' basic information of the two groups was similar (Table 1).

\section{Surgical technique}

All surgeries were performed under general anesthesia by a senior surgeon. For patients in both groups, firstly the fibula fracture was exposed and the fibular rotation

Table 1 Comparison of the baseline data of the patients between group 1 and group 2

\begin{tabular}{lllr}
\hline & Group 1 $(n=20)$ & Group 2 $(n=23)$ & $P$ value \\
\hline Age (years) & $39.60 \pm 14.420$ & $39.22 \pm 13.487$ & 0.929 \\
Gender (male/female) & $12 / 8$ & $13 / 10$ & 0.818 \\
Affected side (left/right) & $7 / 13$ & $9 / 14$ & 0.780 \\
Hospitalization time & $10.20 \pm 2.067$ & $10.09 \pm 1.443$ & 0.835 \\
(days) & & & \\
Follow-up duration & $29.35 \pm 3.617$ & $28.96 \pm 3.994$ & 0.738 \\
(months) & & & 0.604 \\
Mechanism of injury (\%) & & $12(52.2 \%)$ & \\
$\quad$ Road traffic accident & $9(45 \%)$ & $8(34.8 \%)$ & \\
Simple fall & $6(30 \%)$ & $3(13 \%)$ & \\
Sports & $5(25 \%)$ & & \\
\hline
\end{tabular}

Group 1 (ORIF with TEF), and Group 2 (ORIF with DLR), *Independent $t$ test or chi-square test. The $P$ values shown are for inter-group comparisons and length was restored, then an appropriate plate and screws were used to fixed the fibula. When the fragment was larger than $25 \%$ of the articular surface, the posterior malleolar fracture was exposed, reduced and fixed with one or two screws. The syndesmosis was assessed intraoperatively by an external rotation stress test $[16$, 17]. If medial clear space widened more than $5 \mathrm{~mm}$ continuously, syndesmotic fixation was placed.

Next, for patients in group 1, the deltoid ligament was not repaired. Schanz nails were respectively settled into the tibia, the calcaneus and the first metatarsus. The transarticular external apparatus were then installed and adjusted after ORIF, and the ankle was maintained at the neutral position (Fig. 1).

For patients in group 2, after adequate syndesmotic reduction and fixation, an external rotation stress test [11] was performed. The deltoid ligament was repaired if the lateral talar tilt increased or persistent medial space widened more than $5 \mathrm{~mm}$. The ruptured deltoid ligament was exposed, then the superficial and deep layers were respectively repaired with sutures from the suture anchor which was inserted in the medial malleolus or medial talus (Fig. 2).

\section{Postoperative care}

For patients in group 1, they started functional training of the lower extremity soon after operation, and began to do the ankle exercise when the external apparatus was removed at 6 weeks postoperatively. For patients in group 2, the leg was protected in a short plaster cast for 6 weeks. Ankle functional training was initiated when the pain had relieved enough. In group 1, partial weightbearing was instructed at 2 weeks postoperatively, in group 2, this time was 6 weeks. Full weightbearing was allowed when the X-ray film showed sufficient fracture healing.

\section{Outcome assessment}

The pre- and postoperative ankle $\mathrm{X}$-ray films were reviewed to observe fracture healing and diagnose posttraumatic arthritis. We also recorded other complications and patients' inconvenience in daily life. The American Orthopedic Foot and Ankle Society (AOFAS) anklehindfoot score [18], the visual analog scale (VAS) [19], the Medical Outcomes Short Form 36-item questionnaire score (SF-36, [20], and the ankle range of motion (ROM) were used for functional evaluation. To avoid examiner bias, we chose two surgeons who did not participate in all patients' surgeries to perform clinical evaluations and postoperative follow-up.

\section{Statistical analysis}

Statistical analyses were performed with SPSS Statistics 24.0 software (SPSS Inc., Chicago, Illinois). Normality 


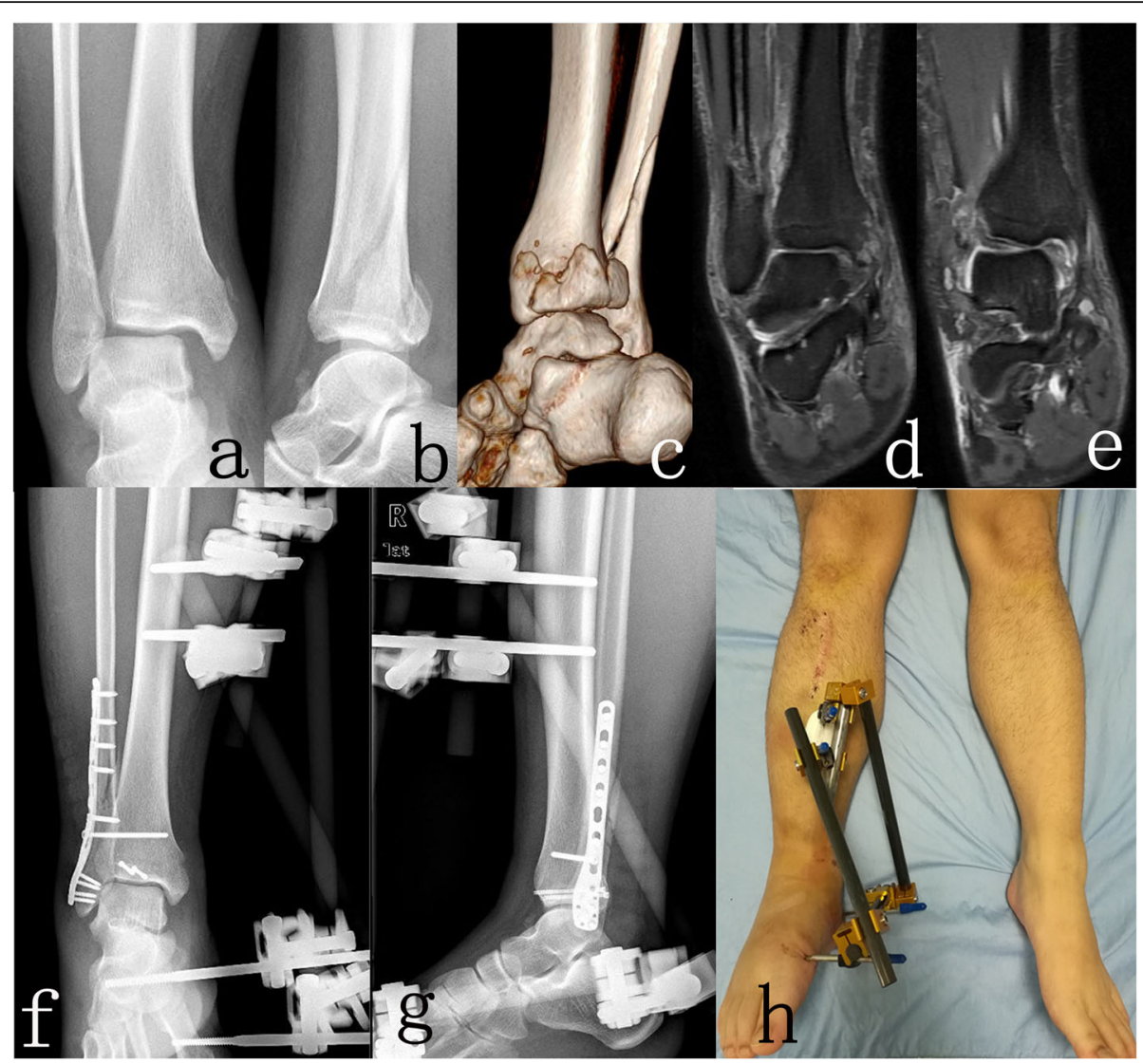

Fig. 1 Imaging studies of a 24 years patient with SER IV ankle fractures (AO/OTA classification 44-B3.1) underwent ORIF with TEF. a-c Preoperative X-rays and three-dimensional CT. $\mathbf{d}$, e Preoperative MRI showing deltoid ligament injury. $\mathbf{f}, \mathbf{g}$ Postoperative anteroposterior and lateral X-rays. $\mathbf{h}$ Postoperative appearance of the affected extremity

was tested using the Kolmogorov-Smirnov test. The chisquare test or Fisher exact test was used to compare categorical data. Differences between groups were evaluated by using the independent $t$ test for normally distributed continuous data and the Mann-Whitney $U$ tests for skewed data. The paired $t$ test was used to compare intra-group continuous variables with normal distribution, and Wilcoxon rank test for continuous variables without normal distribution. All tests were 2-tailed. A $P$ value $<0.05$ was considered statistically significant.

\section{Results}

\section{Baseline characteristics}

The baseline characteristics of the patients were showed in Table 1. 43 patients met all exclusion and inclusion criteria. There were 20 patients in group 1 (8 males [40\%] and 12 females [60\%]) and 23 patients in group 2 (10 males [43.5\%] and 13 females [56.5\%]). With the numbers available, significant difference in patients' age, gender, affected side, hospitalization time and follow-up duration between the two groups was not seen $(P>$ 0.05 ). The main cause was road traffic accident (45 and
$52.2 \%$ respectively), followed by falls (30 and $34.8 \%$ respectively) and sports (5 and 3\%).

\section{Clinical outcomes}

The clinical outcomes between the two groups were listed in Table 2. Patients in group 2 needed longer operative time than patients in group $1(124.5 \pm 6.403 \mathrm{~min}$, $154.78 \pm 8.995 \mathrm{~min}, P<0.001)$. The perioperative blood loss of patients in group 2 was more than that of group $1(81.96 \pm 8.855 \mathrm{ml}, 65.35 \pm 6.385 \mathrm{ml}, \mathrm{P}<0.001)$.

The average AOFAS scores increased significantly in both groups, from $56.45 \pm 4.359$ to $91.30 \pm 3.658$ in group 1, and from $58.04 \pm 3.890$ to $92.13 \pm 3.494$ in group 2. The average VAS scores in both groups decreased significantly, from $5.50 \pm 1.100$ to $1.80 \pm 1.056$, and from $4.96 \pm 1.107$ to $1.70 \pm 1.063$, respectively. The average SF-36 scores in both groups increased significantly, from $57.35 \pm 3.990$ to $78.60 \pm 8.684$, and from $58.09 \pm 4.067$ to $79.57 \pm 7.216$, respectively. Nevertheless, with the numbers available, significant difference for preoperative or postoperative AOFAS, VAS and SF-36 scores between the two groups was not seen $(P>0.05)$. 


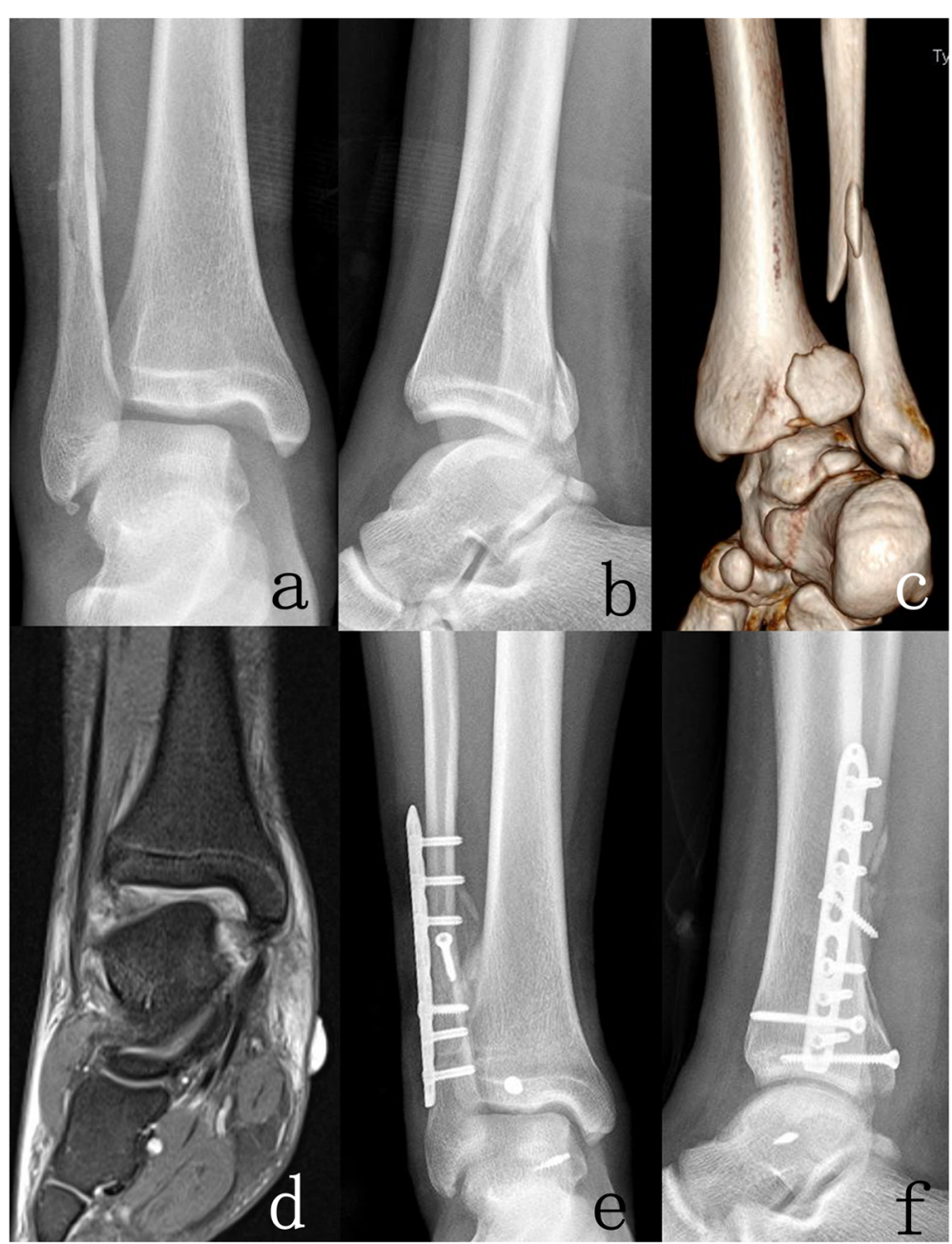

Fig. 2 Imaging studies of a 17 years patient with SER IV ankle fractures (AO/OTA classification 44-B3.1) underwent ORIF with DLR. a-c Preoperative $\mathrm{X}$-rays and three-dimensional CT. $\mathbf{d}$ Preoperative MRI showing deltoid ligament injury. e, $\mathbf{f}$ Postoperative anteroposterior and lateral X-rays

During the follow-up, patients in group 1 spent less time to achieve fracture union $(8.95 \pm 1.986$ weeks, $10.70 \pm 2.548$ weeks, $P=0.017$ ) than patients in group 2 . At 6 weeks postoperatively, the total arc of motion was $29.35 \pm 2.033$ degrees in group 1 compared with $40.35 \pm$ 3.550 degrees in group $2(P<0.001)$. There was no difference between the 2 groups at 12 months postoperatively $(59.65 \pm 3.031$ degrees, $60.43 \pm 3.273$ degrees, $P=$ 0.422 ). In both groups, ankle instability was not seen during follow-ups, no evidences showed bone nonunion or post-traumatic arthritic changes at the last follow-up, delayed union occurred to two patients $(8.7 \%)$ in group 2 . Pin site infection occurred to one patient $(5 \%)$ in group 1 , and two patients (8.7\%) in group 2 appeared superficial wound infections. They were healed after wound dressing. Their main complaints were inconvenience of clothing (17 [85\%] in group 1 and 8 [34.8\%] in group 2) and extremity cleaning (5 [25\%] in group 1 and 19 [82.6\%] in group 2).

\section{Discussion}

The deltoid ligament is an important structure to limit the anterior and posterior translation of the talus and restrain talar abduction, with a relative contribution ranging from 50 to $80 \%[2,21]$.

The acute deltoid ligament injury is commonly associated with ankle joint fractures. There are two most commonly used classification systems, the Lauge-Hansen, and Danis-Weber (AO/OTA) systems. The former relates the suspected mechanism of injury, the latter is based on the location of fibular fracture with respect to syndesmosis. According to the Lauge-Hansen classification, SER IV ankle fractures with an intact medial malleolus represent the ones with a deltoid ligament injury and corresponds to the type B fracture in the DanisWeber classification (AO/OTA classification type 44B3.1). They are one of the most common and unstable fractures in the clinical which need surgical treatment 
Table 2 Comparison of clinical outcomes between group 1 and group 2

\begin{tabular}{|c|c|c|c|}
\hline & Group $1(n=20)$ & Group $2(n=23)$ & $P$ value* \\
\hline Operation time (min) & $124.5 \pm 6.403$ & $154.78 \pm 8.995$ & $<0.001$ \\
\hline Blood loss (ml) & $65.35 \pm 6.385$ & $81.96 \pm 8.855$ & $<0.001$ \\
\hline \multicolumn{4}{|l|}{ AOFAS score } \\
\hline Preoperative & $56.45 \pm 4.359$ & $58.04 \pm 3.890$ & 0.212 \\
\hline Final follow-up & $91.30 \pm 3.658$ & $92.13 \pm 3.494$ & 0.451 \\
\hline \multicolumn{4}{|l|}{ VAS score } \\
\hline Preoperative & $5.50 \pm 1.100$ & $4.96 \pm 1.107$ & 0.115 \\
\hline Final follow-up & $1.80 \pm 1.056$ & $1.70 \pm 1.063$ & 0.749 \\
\hline \multicolumn{4}{|l|}{ SF-36 score } \\
\hline Preoperative & $57.35 \pm 3.990$ & $58.09 \pm 4.067$ & 0.553 \\
\hline Final follow-up & $78.60 \pm 8.684$ & $79.57 \pm 7.216$ & 0.693 \\
\hline Fracture union time (week) & $8.95 \pm 1.986$ & $10.70 \pm 2.548$ & 0.017 \\
\hline Ankle ROM (6 weeks post-op) & $29.35 \pm 2.033$ & $40.35 \pm 3.550$ & $<0.001$ \\
\hline Ankle ROM (12 months post-op) & $59.65 \pm 3.031$ & $60.43 \pm 3.273$ & 0.422 \\
\hline \multicolumn{4}{|l|}{ Complications } \\
\hline Infection & $1(5 \%)$ & $2(8.7 \%)$ & 1.000 \\
\hline Nonunion & $0(0)$ & $0(0)$ & \\
\hline Delayed union & $0(0)$ & $2(8.7 \%)$ & 0.491 \\
\hline Arthritis & $0(0)$ & $0(0)$ & \\
\hline \multicolumn{4}{|l|}{ Inconvenience in daily life } \\
\hline Extremity cleaning & $5(25 \%)$ & 19 (82.6\%) & $<0.001$ \\
\hline Clothing & 17 (85\%) & 8 (34.8\%) & 0.002 \\
\hline
\end{tabular}

$[3,5-9]$. Whether the ruptured deltoid ligament should be repaired or not is still controversy.

Many studies suggested that it was not necessary to repair the ruptured deltoid ligament in ankle fractures [1, 6, 22-25]. In Baird and Jackson's research [22], 24 patients were diagnosed as ankle fractures with the deltoid ligament rupture, 21 patients with no DLR reached a good or excellent rate of $90 \%$, the other 3 were treated with DLR, but 2 of them had unfavorable results. In de Souza's study [23], 22 patients were diagnosed as the deep deltoid ligament rupture, all underwent ORIF and no DLR, all patients had satisfactory outcomes. Stromsoe et al. [24] designed a RCT, symptoms and clinical findings during follow-up showed no differences between two groups. Harper [25] reported 36 patients treated without DLR, the results showed no morbidity or evidence of ligamentous instability, he suggested that when the fibula was anatomic fixed and the medial space was maintained, DLR was not necessary.

However, some studies suggested that repair of the deltoid ligament could reduce long-term complications, patients may develop chronic ankle instability without DLR [7, 21, 25, 26]. Jones and Nunley [7] designed a retrospective comparative study including 27 patients, 12 were treated with lateral malleolus ORIF and DLR, the author concluded that repairing the deltoid ligament at the time of lateral malleolus fixation demonstrates subjective, functional, and radiologic outcomes for bimalleolar equivalent ankle fractures. Zhao et al. [21] reported 74 ankle fractures with deltoid ligament rupture, 20 patients were treated with DLR, this comparative study showed that DLR could decrease the widen medial clear space and malreduction rate. Johnson and Hill [26] reported 30 patients, treated with lateral malleolus ORIF, no DLR, the final outcome was poor in $41 \%$ of them.

As mentioned before, our previous study had shown that ORIF with TEF could achieve satisfactory outcomes in treating SER IV ankle fractures [15]. The present study retrospectively analyzed the results of 43 patients with SER IV ankle fractures who received ORIF with TEF (group 1) or ORIF with DLR (group 2).

For functional recovery and pain relieving, in both groups, AOFAS, SF-36, and VAS achieved satisfactory results after surgery, and with the numbers available, the difference between the two groups was not significant for all the three indexes. As for the total dorsiflexion/ plantarflexion arc of motion, at the time of 6 weeks after surgery, patients in group 2 got significantly improved ankle ROM than patients in group 1 . We considered 
that in the early stage, the ankle was fixed by transarticular external fixator and could not start early exercise, it brought unfavorable ankle functional results. When the fixator was removed after 6 weeks postoperatively, patients in group 1 could start ankle mobilization. Although patients in group 2 toke the exercise much earlier, there was no difference at 12 months postoperatively compared with patients in group 1. No cases of bone nonunion or post-traumatic arthritic changes were seen during the follow-up. So, patients who underwent ORIF with TEF could achieve comparable functional results and pain relieving to those who underwent ORIF with DLR.

According to biomechanical and animal studies, early weight bearing protocols is beneficial that it could optimize fracture healing [27]. For ankle fracture, early weight bearing after ORIF is feasible biomechanically without loss of reduction or fixation failure [28]. Dehghan et al. [16] designed a multicenter RCT, 110 patients with an unstable ankle fracture underwent ORIF, they were randomly divided into two groups, in the early weightbearing group, patients started full weightbearing at 2 week after operation, in the late weightbearing group, patients kept nonweightbearing for 6 weeks. The author recommended early postoperative weightbearing because it brought early improved functional outcome and did not increase complication rate. Papachristou et al. [29] designed a prospective study and suggested that early weight bearing could facilitate recovery and promote fracture union after ORIF for posterior malleolar fracture. In the present study, as ORIF and TEF could provide rigid stability and support, patients in group 1 spent less time to start weightbearing and achieve fracture union.

Both methods brought inconvenience to patients' daily life. A majority of patients in group 2 complained that it was weary to repeat the process of taking on and off the plaster cast when cleaning the affected lower extremity. In group 1, the external fixator provided an open space that did not cover the skin, few patients thought that it was a little difficult to keep the skin clean around the external apparatus. It could also be noticed that most patients in group 1 thought it inconvenient for clothing because special trousers were needed to cope with the external apparatus, few patients in group 2 thought it difficult to wear trousers when the plaster cast was not removed.

There were several limitations in the present study. This was a retrospective study, and it was not randomized when assigning patients to different groups. We just analyzed SER IV fractures without other types of ankle fractures combined with deltoid ligament injury, the sample size was relatively small. Prospective comparative studies involving more samples are necessary for better illustration. However, in our study, the baseline data of the two groups were similar, most patients achieved satisfactory results, this study could provide reference for the management of SER IV fractures which have not been reported before. Regardless of fixation strategy, the ultimate goal is to have patients quickly and painlessly return to their preinjury activities and minimize the incidence of postoperative traumatic arthritis $[5,11,30]$.

\section{Conclusion}

In conclusion, compared with ORIF with DLR, ORIF with TEF but no DLR is an optional strategy to manage SER IV ankle fractures (AO/OTA classification 44-B3.1) as it results in favorable postoperative functional recovery and pain relieving. It also allows patients to start relatively earlier weightbearing and may promote fracture union.

\section{Abbreviations}

AOFAS: American Orthopaedic Foot and Ankle Society; DLR: Deltoid ligament repair; ORIF: Open reduction and internal fixation; ROM: Range of motion; SER: Supination-external rotation; SF-36: Medical Outcomes Short Form 36-item questionnaire; TEF: Transarticular external fixation; VAS: Visual analogue scale

\section{Acknowledgements \\ Not applicable.}

\section{Authors' contributions}

LBH designed the study, analyzed the data, and drafted the manuscript. LL, WSX and ZZD participated in the design of the study and data collection. YH and in LQ collected the data, followup of patients. $L$ helped in writing the manuscript. All authors read and approved the final manuscript.

\section{Funding}

This work was supported by Natural Science Foundation of China (81874002). The funding organization had no role in the design of the study, data collection, analysis, interpretation of data, in writing the manuscript, or decision to publish.

\section{Availability of data and materials}

The data of this study were real and were performed using SPSS Statistics version 24.0 software (SPSS Inc., Chicago, Illinois). All of the data are available in contact with the correspondence author.

\section{Ethics approval and consent to participate}

This study has been approved by the internal research administration department and the ethical committee of West China hospital. The clinical study was performed in accordance with the Declaration of Helsinki on ethical principles for medical research involving human subjects. We have obtained written consent to participate from the participants.

Consent for publication

Not applicable.

\section{Competing interests}

The authors declared no potential conflicts of interest with respect to the research, authorship, and/or publication of this article.

\section{Author details}

'Department of Orthopedics, The First Hospital of Lanzhou University, 1\# West Donggang Road, Lanzhou 730000, People's Republic of China.

${ }^{2}$ Department of Orthopedics, West China Hospital, Sichuan University, 37\#

Wainan Guoxue Road, Chengdu 610041, People's Republic of China. 
Received: 17 June 2019 Accepted: 20 September 2019

Published online: 18 October 2019

\section{References}

1. Kusnezov NA, Eisenstein ED, Diab N, Thabet AM, Abdelgawad A. Medial Malleolar fractures and associated deltoid ligament disruptions: current management controversies. Orthopedics. 2017:40(2):e216-22.

2. Yu GR, Zhang MZ, Aiyer A, Tang X, Xie M, Zeng LR, Zhao YG, Li B, Yang YF. Repair of the acute deltoid ligament complex rupture associated with ankle fractures: a multicenter clinical study. J Foot Ankle Surg. 2015;54(2):198-202.

3. Gougoulias N, Khanna A, Sakellariou A, Maffulli N. Supination-external rotation ankle fractures: stability a key issue. Clin Orthop Relat Res. 2010; 468(1):243-51.

4. Jelinek JA, Porter DA. Management of unstable ankle fractures and syndesmosis injuries in athletes. Foot Ankle Clin. 2009;14(2):277-98.

5. Hintermann B, Regazzoni P, Lampert C, Stutz G, Gachter A. Arthroscopic findings in acute fractures of the ankle. J Bone Joint Surg Br. 2000;82(3):345-51.

6. Michelson JD. Ankle fractures resulting from rotational injuries. J Am Acad Orthop Surg. 2003;11(6):403-12.

7. Jones CR, Nunley JA 2nd. Deltoid ligament repair versus syndesmotic fixation in bimalleolar equivalent ankle fractures. J Orthop Trauma. 2015; 29(5):245-9.

8. Gougoulias N, Sakellariou A. When is a simple fracture of the lateral malleolus not so simple? How to assess stability, which ones to fix and the role of the deltoid ligament. Bone Joint J. 2017;99-B(7):851-5.

9. Wang X, Zhang C, Yin JW, Wang C, Huang JZ, Ma X, Wang CW, Wang X Treatment of medial malleolus or pure deltoid ligament injury in patients with supination-external rotation type IV ankle fractures. Orthop Surg. 2017; 9(1):42-8

10. Hintermann B, Valderrabano V, Boss A, Trouillier HH, Dick W. Medial ankle instability: an exploratory, prospective study of fifty-two cases. Am J Sports Med. 2004;32(1):183-90.

11. Little MM, Berkes MB, Schottel PC, Garner MR, Lazaro LE, Birnbaum JF, Helfet $\mathrm{DL}$, Lorich DG. Anatomic fixation of supination external rotation type IV equivalent ankle fractures. J Orthop Trauma. 2015;29(5):250-5.

12. Lavini F, Maluta T, Carpeggiani G, Dall'Oca C, Samaila E, Marconato G, Magnan B. A new approach to local DCO in ankle fracture dislocations: external fixation with diaphyseal unicortical screws applied by local anaesthesia. Musculoskelet Surg. 2017.

13. Przkora R, Kayser R, Ertel W, Heyde CE. Temporary vertical transarticular-pin fixation of unstable ankle fractures with critical soft tissue conditions. Injury. 2006;37(9):905-8.

14. Van Son MA, De Vries J, Roukema JA, Den Oudsten BL. Health status, health-related quality of life, and quality of life following ankle fractures: a systematic review. Injury. 2013;44(11):1391-402.

15. Li BH, Wang SX, Li J, Huang FG, Xiang Z, Fang Y, Zhong G, Yi M, Zhao XD, Liu L. Early and mid-term results of transarticular external fixation in the treatment of supination-external rotation type IV equivalent ankle fractures. Chin J Traumatol. 2018;21(4):193-6.

16. Dehghan N, McKee MD, Jenkinson RJ, Schemitsch EH, Stas V, Nauth A, Hall JA, Stephen DJ, Kreder HJ. Early Weightbearing and range of motion versus non-Weightbearing and immobilization after open reduction and internal fixation of unstable ankle fractures: a randomized controlled trial. J Orthop Trauma. 2016;30(7):345-52.

17. Sanders DW, Tieszer C, Corbett B. Canadian orthopedic trauma S: operative versus nonoperative treatment of unstable lateral malleolar fractures: a randomized multicenter trial. J Orthop Trauma. 2012;26(3):129-34.

18. Kitaoka HB, Alexander IJ, Adelaar RS, Nunley JA, Myerson MS, Sanders M. Clinical rating systems for the ankle-hindfoot, midfoot, hallux, and lesser toes. Foot Ankle Int. 1994;15(7):349-53.

19. Huskisson EC. Measurement of pain. Lancet. 1974;2(7889):1127-31.

20. Ware JE Jr, Sherbourne CD. The MOS 36-item short-form health survey (SF-36). I. Conceptual framework and item selection. Med Care. 1992; 30(6):473-83.

21. Zhao HM, Lu J, Zhang F, Wen XD, Li Y, Hao DJ, Liang XJ. Surgical treatment of ankle fracture with or without deltoid ligament repair: a comparative study. BMC Musculoskelet Disord. 2017;18(1):543.

22. Baird RA, Jackson ST. Fractures of the distal part of the fibula with associated disruption of the deltoid ligament. Treatment without repair of the deltoid ligament. J Bone Joint Surg Am. 1987;69(9):1346-52.
23. de Souza LJ, Gustilo RB, Meyer TJ. Results of operative treatment of displaced external rotation-abduction fractures of the ankle. J Bone Joint Surg Am. 1985;67(7):1066-74

24. Stromsoe K, Hogevold HE, Skjeldal S, Alho A. The repair of a ruptured deltoid ligament is not necessary in ankle fractures. J Bone Joint Surg Br. 1995;77(6):920-1.

25. Harper MC. The deltoid ligament. An evaluation of need for surgical repair Clin Orthop Relat Res. 1988;226:156-68.

26. Johnson DP, Hill J. Fracture-dislocation of the ankle with rupture of the deltoid ligament. Injury. 1988;19(2):59-61.

27. Kubiak EN, Beebe MJ, North K, Hitchcock R, Potter MQ. Early weight bearing after lower extremity fractures in adults. J Am Acad Orthop Surg. 2013; 21(12):727-38.

28. Tan EW, Sirisreetreerux N, Paez AG, Parks BG, Schon LC, Hasenboehler EA. Early Weightbearing after operatively treated ankle fractures: a biomechanical analysis. Foot Ankle Int. 2016;37(6):652-8.

29. Papachristou G, Efstathopoulos N, Levidiotis C, Chronopoulos E. Early weight bearing after posterior malleolar fractures: an experimental and prospective clinical study. J Foot Ankle Surg. 2003;42(2):99-104.

30. Michelson JD. Fractures about the ankle. J Bone Joint Surg Am. 1995;77(1): 142-52.

\section{Publisher's Note}

Springer Nature remains neutral with regard to jurisdictional claims in published maps and institutional affiliations.
Ready to submit your research? Choose BMC and benefit from:

- fast, convenient online submission

- thorough peer review by experienced researchers in your field

- rapid publication on acceptance

- support for research data, including large and complex data types

- gold Open Access which fosters wider collaboration and increased citations

- maximum visibility for your research: over $100 \mathrm{M}$ website views per year

At BMC, research is always in progress.

Learn more biomedcentral.com/submissions 